\title{
Expression profiles of putative defence-related proteins in oil palm (Elaeis guineensis) colonized by Ganoderma boninense.
}

\begin{abstract}
Basal stem rot (BSR) is a major disease of oil palm caused by a pathogenic fungus, Ganoderma boninense. However, the interaction between the host plant and its pathogen is not well characterized. To better understand the response of oil palm to G. boninense, transcript profiles of eleven putative defence-related genes from oil palm were measured by quantitative reverse-transcription (qRT)-PCR in the roots of oil palms treated with $\mathrm{G}$. boninense from 3 to 12 weeks post infection (wpi). These transcripts encode putative Bowman-Birk serine protease inhibitors (EgBBI1 and 2), defensin (EgDFS), dehydrin (EgDHN), early methionine-labeled polypeptides (EgEMLP1 and 2), glycine-rich RNA binding protein (EgGRRBP), isoflavone reductase (EgIFR), metallothionein-like protein (EgMT), pathogenesis-related-1 protein (EgPRP), and type 2 ribosome-inactivating protein (EgT2RIP). The transcript abundance of EgBBI2 increased in G. boninense-treated roots at 3 and 6. wpi compared to those of controls; while the transcript abundance of EgBBI1, EgDFS, EgEMLP1, EgMT, and EgT2RIP increased in G. boninense-treated roots at 6 or 12. wpi. Meanwhile, the gene expression of EgDHN was up-regulated at all three time points in $\mathrm{G}$. boninense-treated roots. The expression profiles of the eleven transcripts were also studied in leaf samples upon inoculation of $\mathrm{G}$. boninense and Trichoderma harzianum to identify potential biomarkers for early detection of BSR. Two candidate genes (EgEMLP1 and EgMT) that have different profiles in G. boninense-treated leaves compared to those infected by $\mathrm{T}$. harzianum may have the potential to be developed as biomarkers for early detection of G. boninense infection.
\end{abstract}

Keyword: Basal stem rot; Elaeis guineensis; Ganoderma; Oil palm; Real-time PCR. 